\title{
Efficacy Study With Post-Exposure Prophylaxis
}

National Cancer Institute

\section{Source}

National Cancer Institute. Efficacy Study With Post-Exposure Prophylaxis. NCI Thesaurus.

Code C158357.

A study that assesses the efficacy of prophylactic treatment given after exposure to the agent(s) but before the manifestation of the disease or condition. 